\title{
Prescrição de psicofármacos nos cuidados de saúde primários no Porto: estudo transversal
}

Rita Lopes, ${ }^{*}$ John Yaphe, ${ }^{* *}$ Maria José Ribas***

\section{RESUMO}

Objetivo: Caracterizar a prescrição de ansiolíticos e antidepressivos e analisar a associação com características do médico prescritor e da unidade de saúde.

Tipo de estudo: Observacional, analítico, transversal.

Local: Unidades de saúde (US) do Agrupamento de Centros de Saúde Porto Ocidental.

População: Médicos de família.

Métodos: Foram recolhidos dados no Sistema de Informação da Administração Regional de Saúde (SIARS@) que incluíam todas as prescrições efetuadas informaticamente em 2009. Foram analisadas variáveis relativas a cada médico (género, idade, número de utentes e número de unidades ponderadas) e a cada US (tipo e indicadores de morbilidade da população). Estudaram-se os fármacos mais prescritos de cada grupo, alprazolam e fluoxetina, utilizando indicadores preconizados de quantidade (dose diária definida por mil utentes por dia: DUD) e custo. Utilizaram-se medidas descritivas e os testes de qui quadrado, não paramétrico de Kruskal Wallis e correlação de Spearman. O erro alfa aceite como significativo foi de $5 \%$.

Resultados: Os perfis de prescrição de 95 médicos, de 12 US, revelam grande variabilidade (DUD alprazolam: média (M)=19,74, desvio padrão (DP)=9,29, mínimo (Mín)=5,12, máximo (Máx)=60,83; DUD fluoxetina: $M=9,18, D P=4,39, M i ́ n=0,71, M a ́ x=28,37$ ). Os médicos mais prescritores (acima do percentil 95) prescreveram cerca de 5 vezes mais alprazolam do que os menos prescritores (abaixo do percentil 5), o mesmo se verificando para a fluoxetina. A maioria ( $82 \%$ ) prescreve sistematicamente embalagens de 60 comprimidos de alprazolam. Não foram encontradas associações importantes entre os padrões de prescrição e as características avaliadas dos médicos ou das US.

Conclusões: Os resultados são comparáveis a outros estudos, reforçando o potencial do sistema de informação como ferramenta de reflexão acerca da prescrição. A variabilidade encontrada reforça a necessidade de uniformizar os padrões de prescrição entre médicos e de adaptar o sistema de informação à unidade padronizada internacionalmente, o número de DUD, de forma a permitir comparações e medidas de melhoria.

Palavras-chave: Médico de Família; Prescrição; Psicofármaco.

\section{INTRODUÇÃO}

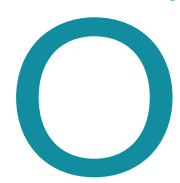

padrão de prescrição de psicofármacos tem vindo a mudar. Questiona-se se o seu uso quase exclusivo na doença mental moderada a grave progrediu para um uso generali-

*Médica Interna de Medicina Geral e Familiar - USF Horizonte, ULS Matosinhos **Professor Associado - Instituto de Ciências da Vida e da Saúde (ICVS), Escola de Ciências da Saúde, Universidade do Minho e ICVS/3B's Laboratório Associado. ***Presidente do Conselho Clínico e de Saúde do ACeS Porto Ocidental Médica Assistente Graduada de Medicina Geral e Familiar - USF Garcia de Orta zado, quiçá imponderado, sobre qualquer manifestação de sofrimento psíquico. ${ }^{1}$ Torna-se, pois, relevante estudar as condicionantes da prescrição destes fármacos.

A variabilidade da prescrição médica na área da psicofarmacologia tem sido estudada em vários países..$^{2-6} \mathrm{~A}$ sua adequação tem sido repetidamente questionada e em múltiplos locais foram identificados problemas com impacto negativo na saúde pública, como: uso de benzodiazepinas como tratamento crónico, prescrição variável de sedativos consoante o tipo de organização da unidade 
de saúde, prescrição excessiva de benzodiazepinas, deficiente diagnóstico e tratamento da depressão., ${ }^{27-11}$

Em 2010, Portugal foi reconhecido como o segundo país do mundo mais consumidor de benzodiazepinas ansiolíticas. ${ }^{12}$ Nos últimos dez anos foram realizados diferentes trabalhos de investigação sobre a utilização de medicamentos em Portugal que permitiram identificar um aumento genérico do consumo de medicamentos na área da patologia mental (benzodiazepinas, antidepressivos e neurolépticos). Vários fatores parecem justificar esta tendência: maior acessibilidade dos doentes às terapêuticas por maior comparticipação no custo das mesmas, desvio da prescrição para alternativas mais recentes e dispendiosas sem benefício clínico evidente e excessiva prescrição (nomeadamente em relação às benzodiazepinas) com afastamento das metas estabelecidas no Plano Nacional de Saúde 2004-2010. ${ }^{13}$

Os médicos de família são os principais prescritores de psicofármacos. ${ }^{2}$ Pelo disposto, torna-se relevante a caracterização da prescrição de psicofármacos a nível dos cuidados de saúde primários (CSP), nomeadamente utilizando as ferramentas informáticas disponíveis. O sistema de prescrição eletrónica, em uso crescente desde 2005 e atualmente generalizado, permite agora um acesso facilitado à prescrição médica. O Sistema de Informação da Administração Regional de Saúde (SIARS, Microstrategy 9) centraliza dados provenientes de vários sistemas de informação, incluindo o Sistema de Apoio ao Médico (SAM) e o Sistema de Informação para as Unidades de Saúde (SINUS). Dada a recente criação dos Agrupamentos de Centros de Saúde (ACeS) e do uso do SIARS a este nível, é importante compreender como pode ser analisada e utilizada a informação que disponibiliza para os profissionais e instituições envolvidas.

A maior parte dos psicofármacos prescritos pertence aos grupos do prontuário terapêutico Antidepressivos (AD) e Ansiolíticos, Sedativos e Hipnóticos (ASH). ${ }^{2}$ Um estudo português identificou a fluoxetina e o alprazolam como os fármacos mais prescritos de cada um destes grupos. ${ }^{14}$

A prescrição é influenciada não só por fatores clínicos, mas também por fatores não clínicos (características intrínsecas do sistema de saúde e do médico e características demográficas, sociais e económicas da população). ${ }^{2}$
Pretende-se analisar a prescrição de psicofármacos no ACeS Porto Ocidental, durante o ano de 2009, destacando a de alprazolam e fluoxetina, utilizando o SIARS, estudando as características disponíveis no sistema acerca da unidade, do médico ou dos utentes que a possam influenciar.

\section{MÉTODOS}

Realizou-se um estudo observacional, analítico, transversal, sobre a prescrição de psicofármacos nas unidades de saúde (US) do ACeS Porto Ocidental, com foco em dois grupos: antidepressivos e ansiolíticos, hipnóticos e sedativos. Da população em estudo - todos os médicos de família do ACeS em atividade profissional entre 1 de janeiro e 31 de dezembro de 2009 - foram excluídos os que se aposentaram ou que não prescreviam eletronicamente $(n=3)$.

A operacionalização de cada variável resultou de um compromisso entre os dados passíveis de obter no SIARS e os indicadores descritos na literatura, que permitem a comparação com outros estudos nacionais e internacionais. ${ }^{15-18}$

\section{Caracterização da prescrição}

As variáveis que caracterizam a prescrição envolvem medidas de volume (número de embalagens, número de comprimidos/cápsulas, número de doses diárias definidas - DDD), de custo e de qualidade (percentagem de embalagens de 60 comprimidos, percentagem do número de DDD de fluoxetina não genéricas). Por simplificação, adiante utilizar-se-á a denominação "número de comprimidos", mesmo que aplicada a fármacos comercializados sob a forma de cápsulas.

\section{Volume}

As medidas de volume utilizadas (número de embalagens, número de comprimidos, número de DDD) estão descritas nas Guidelines for ATC Classification and DDD Assignment do WHO Collaborating Centre for Drug Statistics Methodology.$^{18}$ O SIARS disponibiliza informação relativa ao número de embalagens prescrito. Através da informação sobre a composição de cada embalagem, é possível determinar manualmente o número de comprimidos prescrito. No entanto, a variedade existente de doses e tamanhos de embalagens para cada substância ativa limita o uso destas medidas 
na comparação da prescrição. Só ao nível das substâncias ativas mais prescritas (alprazolam e fluoxetina) é que foi obtida a medida recomendada pela Organização Mundial de Saúde, o número de DDD prescrito. ${ }^{18} \mathrm{~A}$ DDD representa a dose de manutenção diária média de adultos para a principal indicação da substância ativa, por via de administração. A DDD descrita, para a via oral, para as substâncias ativas estudadas é de $20 \mathrm{mg}$ para a fluoxetina e de $1 \mathrm{mg}$ para o alprazolam. Através do número de comprimidos e da dose de cada embalagem foi possível calcular o número de DDD prescrito. Este parâmetro permite comparações entre populações, independentemente das diferenças de preço, moeda, tamanhos de embalagem e dosagens, que variam de país para país.

A medida "número de DDD/1.000utentes/dia" (DUD) resulta de uma adaptação da medida preconizada "número de DDD/1.000habitantes/dia" (DID). ${ }^{17}$ É obtida a partir do número de DDD prescritas e do número de utentes de cada médico, segundo a fórmula:

DUD = número de DDD/número de utentes x 1.000/365

Por exemplo, um médico que prescreva 12.410 DDD de alprazolam por ano (que podem corresponder a 12.410 comprimidos de $1 \mathrm{mg}$ de alprazolam ou a 24.820 comprimidos de $0,5 \mathrm{mg}$ de alprazolam, etc.), a uma lista de 1.700 utentes, prescreve 20 DUD. Este número significa, em estimativa, que 20 em cada 1.000 utentes do médico estiveram em tratamento com alprazolam durante o ano. A DUD que prescreveu é então comparável à DUD prescrita por outros médicos de Portugal e outros países.

\section{Custo}

As variáveis de custo foram definidas de acordo com as recomendações do projeto EUROMEDSTAT (Statistics on Medicines in Europe). ${ }^{17} \mathrm{O}$ SIARS disponibiliza dados sobre o Preço de Venda ao Público (PVP) e sobre o custo para o SNS (Serviço Nacional de Saúde) de cada embalagem prescrita. Estes dados foram trabalhados de acordo com o número de DDD a que correspondem.

\section{Possíveis determinantes}

Sabendo-se que a prescrição médica é influenciada por fatores não clínicos, neste estudo pretende-se ana- lisar a relação entre as características da unidade de saúde, médico e população e a prescrição.

Espera-se que a prescrição seja influenciada pelo número de utentes inscritos e pela estrutura etária da população, sendo que esta aumenta com o aumento da idade, tendo em conta os utentes que efetivamente utilizaram os serviços em 2009 (através da taxa de utilização de consultas de cada unidade). ${ }^{7,23} \mathrm{O}$ número de Unidades Ponderadas (UP) da lista de um médico pode ter implicações na sua carga assistencial, qualidade de cuidados e prescrição. As patologias inerentes à prescrição dos dois subgrupos de psicofármacos em estudo revelam-se mais na população feminina, o que justificará uma eventual relação entre o número de utentes do sexo feminino de uma unidade e o número de prescrições..$^{21}$ Sabe-se também que a depressão é mais frequente em populações com comorbilidades, tendo sido analisados o número de diabéticos e o número de pessoas isentas de taxas moderadoras por declaração médica definitiva. ${ }^{7}$ É ainda reconhecido que a prevalência de doenças psiquiátricas é superior em populações com carências económicas, motivo pelo qual foi avaliado o número de doentes isentos de taxa moderadora por motivos económicos. ${ }^{7}$ Uma unidade de saúde que tenha muitos utentes sem médico de família atribuído poderá prestar cuidados menos personalizados e contínuos, o que poderá influenciar a prescrição. ${ }^{2}$ A idade e o género dos prescritores têm sido implicados nos comportamentos de prescrição. ${ }^{23}$

\section{Recolha dos dados}

Utilizaram-se dados secundários anonimizados retirados do SIARS, sob autorização da direção do ACeS. O programa permite que cada prescritor seja apresentado primariamente por um código, sem o seu nome associado. A idade e o género de cada médico foram fornecidos pelo ACeS, a partir da lista de códigos de cada prescritor. Os dados foram selecionados, compilados e trabalhados no programa Microsoft Office Excel $2007^{\circledR}$.

\section{Análise Estatística}

A análise estatística foi realizada com recurso ao software EpiInfo ${ }^{T M}$, versão 3.5.1. A análise univariável foi utilizada para medidas descritivas (frequência absoluta, frequência relativa, média, desvio-padrão [DP], mínimo [Mín] e máximo [Máx]).

A análise bivariável foi efetuada para testar as asso- 
ciações entre variáveis de diferentes tipos. Entre variáveis qualitativas nominais, como o género e o tipo de unidade, foi utilizado o teste do qui-quadrado. Entre variáveis quantitativas contínuas e qualitativas, como o número de DDD e o tipo de unidade, foi utilizado o teste não paramétrico de Kruskal-Wallis, uma vez que o pressuposto da normalidade não estava cumprido para todas as variáveis quantitativas. A relação entre variáveis quantitativas foi testada através da correlação de Spearman. $\mathrm{O}$ nível de significância foi estabelecido em 0,05 .

O protocolo do estudo foi aprovado pela Comissão de Ética para a Saúde da Administração Regional de Saúde do Norte.

\section{RESULTADOS}

A população de prescritores em estudo é composta por 95 médicos de família, com idade mediana de 55 anos, sendo $72 \%$ do género feminino. Cerca de $70 \%$ trabalham em oito Unidades de Cuidados de Saúde Personalizados (UCSP) e os restantes em quatro Unidades de Saúde Familiar (USF), sendo responsáveis por 193.795 utentes. O número médio de utentes por médico é de 1.684 e varia entre 1.102 e 2.167 .

As unidades de saúde são distintas na dimensão (dois a 13 médicos por unidade), na percentagem de utentes sem médico ( 0 a $36 \%$ ), na taxa de utilização (37\% a 66\%) e na percentagem de consultas pelo próprio médico ( $67 \%$ a $92 \%$ ). Cada unidade tem também diferente percentagem de utentes com diabetes e com isenção, indicadores de morbilidade populacional.

Em 2009, os psicofármacos corresponderam a 14,3\% das embalagens e a $8,4 \%$ dos custos totais de fármacos prescritos no ACeS.

No grupo dos antidepressivos (21\% das embalagens e $51 \%$ dos custos), os inibidores seletivos da recaptação da serotonina representam $59 \%$ da prescrição e a fluoxetina é o fármaco mais prescrito (cerca de $20 \%$ de todos os $\mathrm{AD})$.

\begin{tabular}{|c|c|c|c|c|c|c|}
\hline \multicolumn{7}{|c|}{$\begin{array}{l}\text { QUADRO I. Características dos médicos de família e respetivas } \\
\text { listas de utentes, de um ACeS, no ano de } 2009 \text {, no contexto do } \\
\text { estudo da prescrição de psicofármacos }(n=95)\end{array}$} \\
\hline & & Número & Média & DP & Mín & Máx \\
\hline \multirow[t]{2}{*}{ Género } & Masculino & 27 & & & & \\
\hline & Feminino & 68 & & & & \\
\hline \multirow[t]{2}{*}{ Idade } & Masculino & & 54 & 6 & 33 & 60 \\
\hline & Feminino & & 53 & 6 & 30 & 61 \\
\hline \multirow[t]{2}{*}{ Unidade de saúde } & USF & 29 & & & & \\
\hline & UCSP & 66 & & & & \\
\hline \multicolumn{2}{|c|}{ Número de utentes da lista } & & 1.684 & 168 & 1.102 & 2.167 \\
\hline \multicolumn{2}{|c|}{$\begin{array}{l}\text { Número de unidades } \\
\text { ponderadas da lista }\end{array}$} & & 2.129 & 210 & 1.452 & 2.620 \\
\hline
\end{tabular}

QUADRO II. Características das unidades de saúde de um ACeS, em 2009 no contexto do estudo da prescrição de psicofármacos $(n=12)$

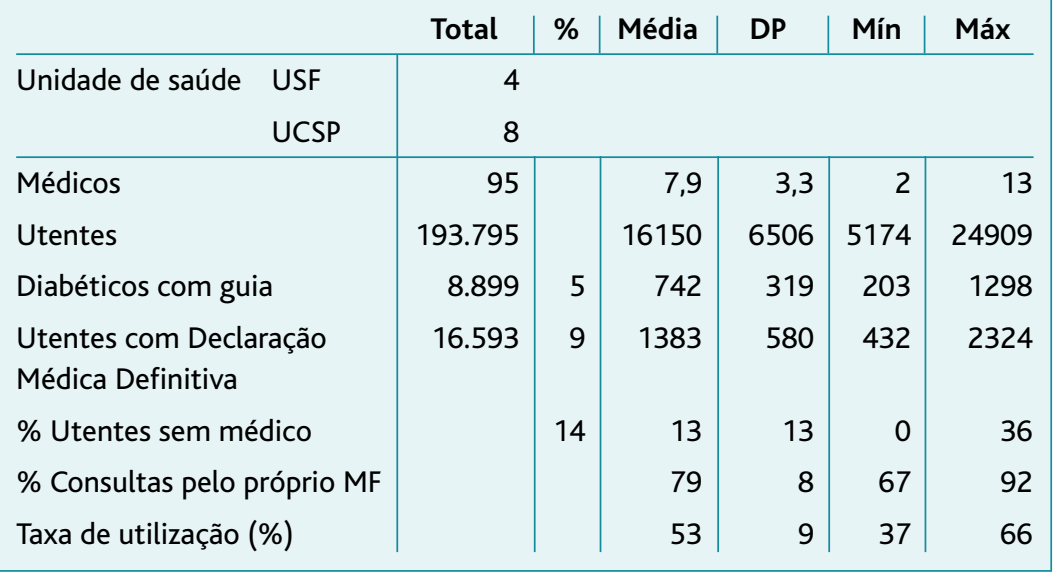

No grupo dos ansiolíticos, sedativos e hipnóticos ( $74 \%$ das embalagens e $33 \%$ dos custos), as benzodiazepinas compõem $90 \%$ da prescrição, sendo o alprazolam o fármaco mais prescrito (cerca de $24 \%$ de todos os ASH).

A DUD média de alprazolam prescrita foi de 19,74 DDD/1.000utentes/dia. O grupo dos mais prescritores (acima do percentil 95) prescreve cerca de 4,6 vezes mais do que os menos prescritores (abaixo do percentil 5). A razão entre o maior e o menor prescritor é de 12. Todos os médicos prescrevem predominantemente embalagens de 60 comprimidos de alprazolam (Mín $57 \%$, Média 83\%, DP 10\%) e alguns não prescrevem em- 
QUADRO III. Características da prescrição de Alprazolam e de Fluoxetina, num ACeS, em 2009, no contexto do estudo da prescrição de psicofármacos

\begin{tabular}{|c|c|c|c|c|c|c|}
\hline & \multirow{2}{*}{$\begin{array}{c}\text { Total } \\
\text { Frequência }\end{array}$} & \multicolumn{4}{|c|}{ Por médico } \\
\hline & & & Média & DP & Mín & Máx \\
\hline & Número de DDD & 1.147 .049 & 12074 & 5545 & 2990 & 32925 \\
\hline$\frac{E}{0}$ & DUD & & 19,74 & 9,29 & 5,12 & 60,83 \\
\hline $\begin{array}{l}\frac{N}{0} \\
\frac{0}{2}\end{array}$ & $\begin{array}{l}\% \text { embalagens de } \\
60 \text { comprimidos }\end{array}$ & & 83 & 10 & 57 & 100 \\
\hline & $\mathrm{PVP} /$ número de $\mathrm{DDD} €$ & & 0,17 & 0,02 & 0,12 & 0,22 \\
\hline & Número de DDD & 537.372 & 5657 & 2831 & 416 & 16216 \\
\hline 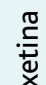 & $\begin{array}{l}\text { \% número de DDD } \\
\text { não genéricos }\end{array}$ & & 27 & 17 & 0 & 76 \\
\hline$\frac{0}{\vec{Z}}$ & DUD & & 9,18 & 4,39 & 0,71 & 28,37 \\
\hline & $\mathrm{PVP} /$ número de $\mathrm{DDD} €$ & & 0,34 & 0,02 & 0,28 & 0,42 \\
\hline
\end{tabular}

dade intra-unidade de saúde, demonstrada pelo efeito gráfico em escada ( $\mathrm{Fi}$ gura 1). A razão entre a unidade de saúde mais prescritora e a menos prescritora é de 2,3 .

A prescrição média de fluoxetina foi de 9,18 DUD. Os mais prescritores prescrevem cerca de 4,7 vezes mais do que os menos prescritores. Alguns médicos só prescrevem genéricos, mas em média $27 \%$ do número de DDD de fluoxetina provêm de medicamentos não genéricos, o que faz variar o preço diário do tratamento de $0,28 €$ até $0,42 €$ consoante a escolha do médico/pedido do utente (Quadro III). Verifica-se o mesmo efeito em escada na prescrição de fluoxetina, dentro de cada unidade (Figura 2). A razão entre a unidade mais prescritora e a menos prescritora é de 2,2.

A correlação de Spearman foi aplicada para determinar as relações entre a prescrição de psicofármacos
Ao agrupar a prescrição de alprazolam por US na qual trabalha cada prescritor verifica-se uma variabili-

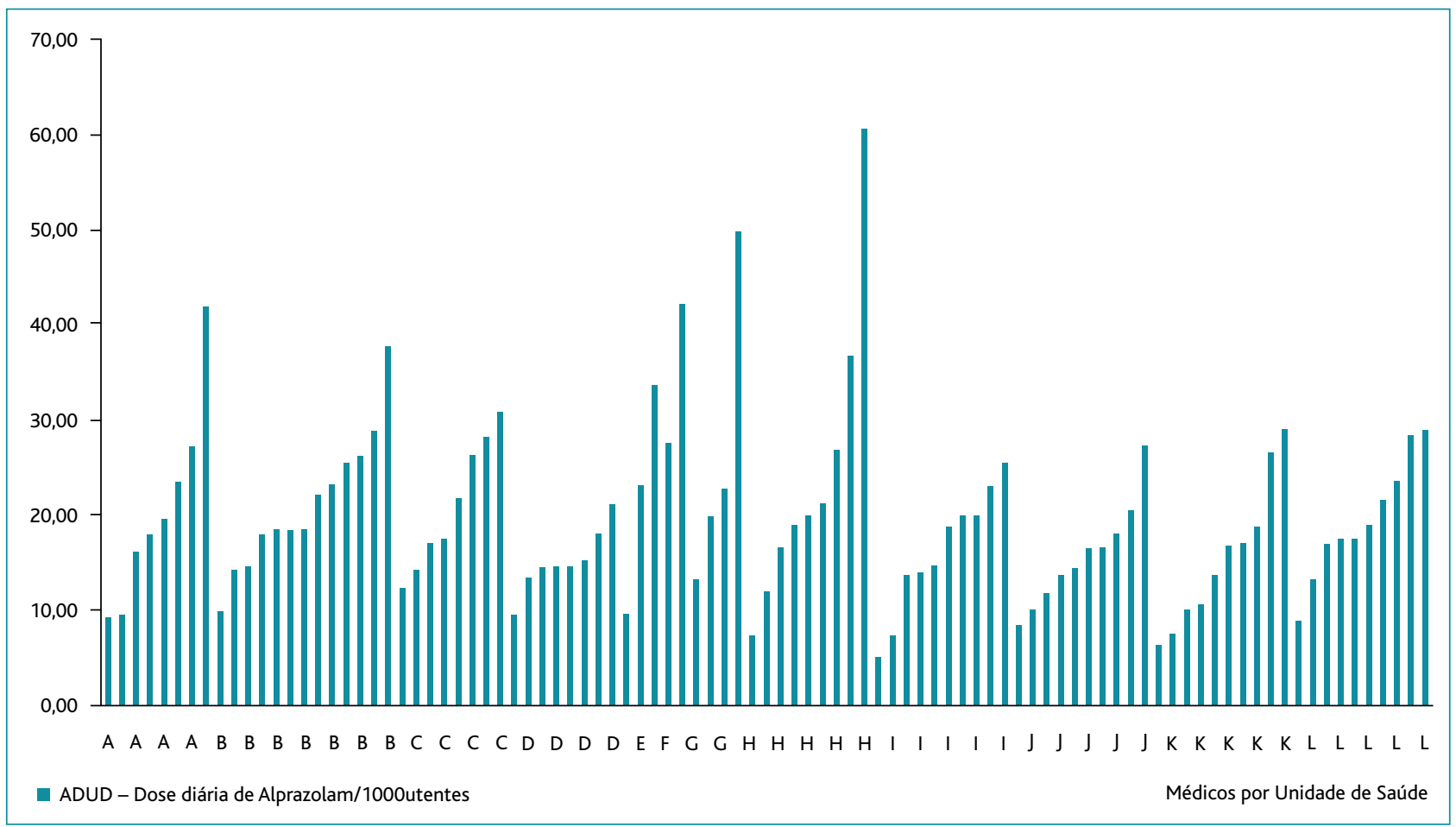

Figura 1. DUD de Alprazolam por médico $(n=95)$ e unidade de saúde $(n=12)$, num ACeS, em 2009, no contexto do estudo da prescrição de psicofármacos. Cada barra representa um médico que trabalha numa das 12 unidades de saúde, representadas pelas letras $A$ até $\mathrm{L}$. 


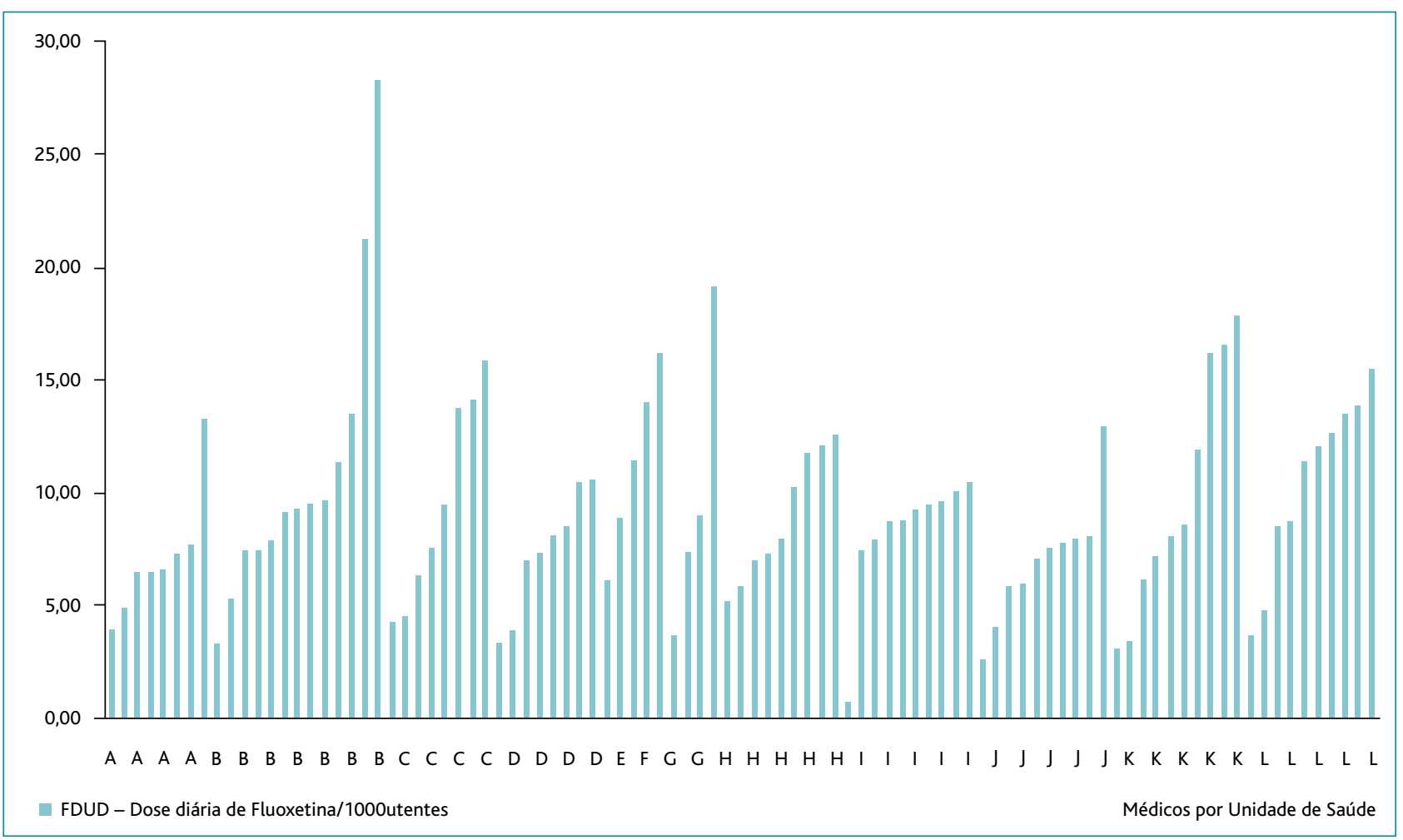

Figura 2. DUD de Fluoxetina por médico $(n=95)$ e unidade de saúde $(n=12)$, num ACeS, em 2009, no contexto do estudo da prescrição de psicofármacos. Cada barra representa um médico que trabalha numa das 12 unidades de saúde, representadas pelas letras $A$ até L.

e o tamanho da lista, a sua ponderação, a percentagem de mulheres.

Na análise de Spearman verificou-se uma associação fraca mas significativa entre o número de DDD de fluoxetina prescritas e o número de unidades ponderadas ( $r s=0,218, p=0,034)$ e o número de utentes mulheres $(r s=0,232, p=0,018)$ da lista. No entanto, o número diário de DDD/1.000 utentes não manteve esta associação significativa com o número de utentes de cada lista.

O número de DDD e o número diário de DDD/ 1.000 utentes de alprazolam prescritas não demonstrou associações significativas.

A relação entre a prescrição de alprazolam e a prescrição de fluoxetina também foi testada com a correlação de Spearman. Verificou-se uma relação positiva entre as duas variáveis que foi estatisticamente significativa $(r s=0,500, p=0,01)$.

\section{DISCUSSÃO}

Este estudo de prescrição de psicofármacos nos cui- dados de saúde primários, realizado numa área urbana de Portugal, revela grande variabilidade na prescrição de alprazolam e fluoxetina entre médicos de família e entre unidades de saúde. A análise das características dos médicos prescritores, utentes e US não permitiu compreender o que condiciona estas diferenças.

O estudo revela ainda que os psicofármacos mais prescritos são a fluoxetina e o alprazolam, resultados que coincidem com os descritos na literatura nacional. ${ }^{13} \mathrm{Na}$ literatura internacional analisada verifica-se que os $\mathrm{AD}$ mais prescritos também pertencem ao grupo dos inibidores seletivos da recaptação da serotonina e são a sertralina (Austrália, Lituânia), paroxetina (Canadá, Espanha) e a fluoxetina (Chile). ${ }^{3,5-6,24}$ Noutros países, as benzodiazepinas mais prescritas são o diazepam (Sérvia e Montenegro, Austrália) e o lorazepam (Canadá). ${ }^{25-26}$

Pouco se pode inferir acerca da qualidade da prescrição a nível populacional sem informações sobre o motivo da prescrição e a prevalência da(s) doença(s) as- 
sociada(s).$^{15}$ A codificação de diagnósticos no SAM não ocorre ainda de modo uniforme, pelo que não se pode relacionar a prescrição de fármacos com a prevalência de patologias baseada na codificação.

A DUD média de alprazolam prescrita no ACeS em $2009(19,74)$ aproxima-se da DID dispensada a nível nacional em $2001(20,52)$, apesar dos diferentes denominadores populacionais. ${ }^{10}$ Isto parece indicar que a metodologia de utilização do SIARS definida neste estudo para a obtenção das DUD é apropriada.

Apesar de semelhante à DID nacional, esta DUD média é de questionável adequação clínica. Por um lado, porque Portugal foi alertado, já em 2004, pelo International Narcotics Control Board para a necessidade de rever o sistema de controlo de distribuição bem como as práticas de prescrição de benzodiazepinas ansiolíticas, dado que mundialmente era já um dos maiores consumidores destas (93,66 DID) ${ }^{9,12}$ Desde então, a tendência identificada agravou-se e os dados mais recentes, do relatório de 2013, demonstram uma utilização de 117,98 DID de benzodiazepinas ansiolíticas. Por outro, porque este estudo demonstra que a prescrição de embalagens de 60 comprimidos é predominante, o que pode indicar uma prescrição destes fármacos tendencialmente para períodos de tempo mais longos do que o máximo agora recomendado pela Norma. ${ }^{19-22}$

No SIARS estão também disponíveis dados acerca das embalagens dispensadas nas farmácias, prescritas pelos médicos do ACeS em 2009, que demonstram ainda maior percentagem de embalagens de 60 comprimidos adquiridas. A prescrição de embalagens de 40 comprimidos, muitas com Autorização de Introdução no Mercado mas ainda não disponíveis neste, induz ao fornecimento de embalagens de 60 comprimidos, de acordo com as regras de substituição de fármacos.

Não foram encontrados estudos portugueses publicados relativamente ao número de DDD de fluoxetina prescrito/dispensado. Dos dados internacionais disponíveis, um estudo australiano de 2005 reporta 6,08 DID de fluoxetina dispensadas, de um total de 51,50 DID de antidepressivos prescritos. ${ }^{3}$ Num estudo espanhol de 2004 foram dispensadas 6,88 DID de fluoxetina e 43,5 DID de antidepressivos. ${ }^{24}$

Uma DUD média de fluoxetina prescrita de 9,18 DDD/1.000utentes/dia pode exprimir, com algumas reservas, que quase $1 \%$ dos utentes de cada médico to- mam 1 cápsula de fluoxetina de $20 \mathrm{mg}$ por dia ao longo do ano. ${ }^{10,16}$ Assumindo a prevalência de depressão descrita para a Europa de 5\%, em conjunto com o facto de a fluoxetina corresponder a cerca de $1 / 5$ das embalagens de todos os $\mathrm{AD}$ prescritos, é possível que esta $\mathrm{DUD}$ média seja adequada, não indiciando sub nem sobre tratamento da depressão.

As associações significativas verificadas entre o número de DDD de fluoxetina e o número de utentes, de unidades ponderadas e de mulheres eram expectáveis: os médicos com maiores listas terão mais utentes em tratamento. Já o número diário de DDD/1.000 utentes de fluoxetina prescritas não manteve esta associação significativa, o que indicaria uma maior tendência de prescrição dos médicos com mais utentes.

Uma limitação deste estudo consiste na não avaliação de outros motivos que possam alterar a prescrição por ausência programada do médico, podendo a DUD de alprazolam e fluoxetina de alguns prescritores estar subestimada ou sobrestimada. Isto poderá ser resolvido pela realização de uma análise da assiduidade concomitante, capaz de identificar prescritores sujeitos a circunstâncias excecionais.

A avaliação da prescrição e não da faturação dos fármacos pode sobrestimar a real utilização dos mesmos pelos utentes, não sendo possível contabilizar com o SIARS, por exemplo, receitas duplicadas ou não adesão ao tratamento. No entanto, a avaliação da prescrição é um melhor indicador das decisões dos prescritores. Poderá ter interesse caracterizar tanto a prescrição como a faturação em estudos subsequentes.

As características das listas estudadas foram insuficientes para justificar a diversidade de prescrição de alprazolam e fluoxetina entre médicos da mesma unidade de saúde, o que salienta a importância da caracterização de variáveis intrínsecas aos valores, crenças, atitudes, experiência e conhecimento dos médicos. Estudos posteriores deverão também ter em conta esta dimensão. ${ }^{2}$

Os estudos farmacoepidemiológicos estão a adquirir cada vez maior dimensão e importância em vários países, consequência da evolução e integração de bases de dados. ${ }^{27} \mathrm{O}$ potencial do SIARS para caracterizar, compreender e fundamentar intervenções sobre a prescrição médica deve continuar a ser explorado e melhorado. A necessidade de introdução manual do nú- 
mero de DDD correspondente a cada dose e nome comercial da substância ativa, já que Portugal é um dos países da Europa com elevada razão número de nomes comerciais/número de substâncias ativas (11,67; mínimo europeu de 2,68), não permitiu o estudo de mais fármacos neste estudo. Seria ideal reestruturar o SIARS de forma a possibilitar o acesso às prescrições em número de DDD e não só ao número de embalagens.

É pertinente continuar a trabalhar os dados existentes para expandir esta análise às restantes substâncias ativas das classes mais prescritas, o que poderá fortalecer a discussão da adequação da prescrição e permitir uma intervenção junto dos prescritores. Por exemplo, é considerado sinal de prescrição inadequada o uso de muitas benzodiazepinas diferentes para justificar $90 \%$ do número de DDD prescritas, doravante designada por DU $90 \% .{ }^{16}$ Neste estudo, esta medida adaptada de modo grosseiro (baseada no número de comprimidos prescritos e não no número de DDD) permite concluir que são necessárias oito benzodiazepinas diferentes para justificar a prescrição de $90 \%$ dos comprimidos. Esta diversidade de prescrição de benzodiazepinas para atingir a DU 90\% é sobreponível à do Canadá, onde também oito foram necessárias. Já na Austrália, a DU 90\% é atingida com quatro benzodiazepinas, talvez devido ao reembolso de somente cinco tipos de benzodiazepinas. ${ }^{25}$ Noutros países, como a Inglaterra, onde se utilizam formulários de prescrição, este parâmetro é também mais baixo.

Concluindo, este estudo utiliza uma metodologia que explora o potencial do SIARS como ferramenta de reflexão acerca da prescrição médica, agora acessível à maioria dos médicos de família. A variabilidade encontrada intra e inter-unidades de saúde de uma mesma área geográfica reforça a necessidade de adaptar o SIARS à unidade padronizada internacionalmente, o número de DDD, de forma a permitir comparações consequentes e identificar eventuais necessidades de formação.

O consumo de benzodiazepinas em Portugal tem aumentado e mantém-se dos mais elevados a nível mundial, sendo provavelmente a sua prescrição excessiva e inapropriada.

\section{AGRADECIMENTOS}

Ao Patrício Costa e Pedro Teixeira pelo seu apoio estatístico.

\section{REFERÊNCIAS BIBLIOGRÁFICAS}

1. Sim MG, Khong E, Wain TD. The prescribing dilemma of benzodiazepines. Aust Fam Physician. 2007;36(11):923-6.

2. Linden M, Lecrubier Y, Bellantuono C, Benkert O, Kisely S, Simon G. The prescribing of psychotropic drugs by primary care physicians: an international collaborative study. J Clin Psychopharmacol. 1999;19(2): 132-40.

3. Smith AJ, Sketris I, Cooke C, Gardner D, Kisely S, Tett SE. A comparison of antidepressant use in Nova Scotia, Canada and Australia. Pharmacoepidemiol Drug Saf. 2008;17(7):697-706.

4. Smith A, Sketris I, Cooke C, Gardner D, Kisely S, Tett S. A comparison of benzodiazepines and related drug use in Nova Scotia, Canada and Australia. Can J Psychiat. 2008;53(8):545-52.

5. Kaduševičius E, Mikučionytè E, Mačiulaitis R, Milvidaitè I, Sveikata A. Trends in the consumption of antidepressant drugs in Lithuania in 2002-2004. Medicina (Kaunas). 2006;42(12):1020-9.

6. Jiron M, Machado M, Ruiz I. Consumo de antidepresivos en Chile entre 1992 y 2004 [Consumption of antidepressants in Chile from 1992 to 2004]. Rev Med Chile. 2008;136(9):1147-54. Spanish

7. Sartorius N, Baghai TC, Baldwin DS, Barrett B, Brand U, Fleischhacker $W$, et al. Antidepressant medications and other treatments of depressive disorders: a CINP Task Force report based on a review of evidence. Int J Neuropsychopharmacol. 2007;10 Suppl 1:S1-207.

8. Furtado C, Teixeira I. Utilização de benzodiazepinas em Portugal continental (1999-2003) [Benzodiazepine's utilization in Continental Portugal (1999.2003)]. Acta Med Port. 2006;19(3):239-46. Portuguese

9. International Narcotic Control Board. Report of the INCB for 2004. New York: United Nations; 2004.

10. António A, Remísio E. Evolução do consumo de benzodiazepinas em Portugal de 1995 a 2001. Lisboa: Observatório do Medicamento e dos Produtos de Saúde, INFARMED; 2002.

11. Ribeirinho M, Monteiro C, Oliveira R. Benzodiazepines's utilization and forecast in Portugal Mainland. Lisbon: INFARMED; 2009 [cited 2014 Dec 7]. Available from: http://www.infarmed.pt/portal/page/portal/INFARMED/MONITORIZACAO_DO_MERCADO/OBSERVATORIO/INTRODUCAO_DE_FICHEIROS/ISPE_OMPS_BZDs.pdf

12. International Narcotic Control Board. Psychotropic substances: report for 2011. New York: United Nations; 2012.

13. Vaz AF, Pinto CG, Lourenço A, Monteiro E, Barros H, Vale, MC, et al. Política do medicamento, dispositivos médicos e avaliação de tecnologias em saúde: plano nacional de saúde 2011-2016. Lisboa: Alto Comissariado da Saúde; 2010 [cited 2014 Dec 7]. Available from: http://pns.dgs.pt/files/2010/11/PM1.pdf

14. Falcão IM, Monsanto A, Nunes B, Marau J, Falcão JM. Prescrição de psicofármacos em medicina geral e familiar: um estudo na rede de médicos-sentinela. Rev Port Clin Geral. 2007;23(1):17-30. Portuguese

15. de Vries CS, Tromp TF, Blijleven W, de Jong-van den Berg LT. Prescription data as a tool in pharmacotherapy audit (I). Pharm World Sci. 1999;21(2):80-4.

16. EURO-MED-STAT. The library of European Union pharmaceutical indicators: expenditure and utilisation indicators. Brussels: EURO-MEDSTAT; 2004 [cited 2014 Dec 7]. Available from: http://ec.europa.eu/ health/ph_projects/2001/monitoring/fp_monitoring_2001_frep_12_3 _en.pdf 
17. EURO-MED-STAT. The library of European Union pharmaceutical indicators: price indicators. Brussels: EURO-MED-STATS; 2004 [cited 2014 Dec 7]. Available from: http://ec.europa.eu/health/ph_projects/2001/ monitoring/fp_monitoring_2001_frep_12_2_en.pdf

18. WHO Collaborating Centre for Drug Statistics Methodology. Anatomical therapeutic chemical (ATC) classification index including defined daily doses (DDD) for plain substances. Geneva: WHO; 2004 [cited 2014 Dec 7]. Available from: http://www.whocc.no/atcddd/

19. World Health Organization. Programme on substance abuse: rational use of benzodiazepines. Copenhagen:WHO; 1996.

20. Longo LP, Johnson B. Addiction: part I. Benzodiazepines: side effects, abuse risk and alternatives. Am Fam Physician. 2000;61(7):2121-8.

21. Longo LP, Parran T, Johnson B, Kinsey W. Addiction: part II. Identification and management of the drug-seeking patient. Am Fam Physician. 2000;61(8):2401-8.

22. Nelson J, Chouinard G. Guidelines for the clinical use of benzodiazepines: pharmacokinetics, dependency, rebound and withdrawal. Can J ClinPharmacol. 1999;6(2):69-83.

23. Orzella L, Chini F, Giorgi Rossi P, Borgia P. Physician and patient characteristics associated with prescriptions and cost of drugs in the Lazio region of Italy. Health Policy. 2010;95(2-3):236-44.

24. Ubeda A, Cardo E, Sellés N, Broseta R, Trillo JL, Fernández-Llimós F. An- tidepressant utilization in primary care in a Spanish region: impact of generic and reference-based pricing policy (2000-2004). Soc Psychiatry Psychiatr Epidemiol. 2007;42(3):181-8.

25. Smith AJ, Sketris I, Cooke C, Gardner D, Kisely S, Tett SE. A comparison of benzodiazepine and related drug use in Nova Scotia and Australia. Can J Psychiatry. 2008;53(8):545-52.

26. Divac N, Tosevski DL, Babic D, Djuric D, Prostran M, Samardzic R. Trends in consumption of psychiatric drugs in Serbia and Montenegro 2000-2004. Pharmacoepidemiol Drug Saf. 2006;15(11):835-8.

27. Furu K, Wettermark B, Andersen M, Martikainen JE, Almarsdottir AB, Sörensen HT. The Nordic Countries as a cohort for pharmacoepidemiological research. Basic Clin Pharmacol Toxicol. 2010;106(2):86-94.

\section{CONFLITO DE INTERESSES}

Inexistentes. John Yaphe é membro do corpo editorial da RPMGF, mas não participou em qualquer decisão editorial sobre este artigo.

\section{ENDEREÇO PARA CORRESPONDÊNCIA}

yonahyaphe@hotmail.com

Recebido em 05-04-2014

Aceite para publicação em 02-10-2014

\section{ABSTRACT}

\section{PSYCHOTROPIC MEDICATION PRESCRIBING IN PRIMARY CARE IN PORTO: A CROSS-SECTIONAL STUDY}

Purpose: To characterize antidepressant and anxiolytic drug prescribing in primary care in Porto using a prescription registry and to analyze the variables that may be associated with psychotropic prescription.

Study design: Cross-sectional

Setting: Primary health care units, West Porto, Portugal.

Participants: Family physicians.

Methods: Data was collected using the SIARS@ database, including all electronic prescriptions issued in primary care in 2009. Variables analyzed related to the physician (gender, age, number of patients and number of weighted patient units) and the health unit (type and population morbidity indicators, patients without a permanent doctor). The most frequently prescribed drugs in each group, fluoxetine and alprazolam, were studied by amount prescribed (defined daily dose per thousand patients per day - DUD) and costs. Descriptive analysis, qui-square, non-parametric Kruskal-Wallis tests and Spearman correlation were used. Significance level was set at $p<0.05$.

Results: There were no associations found between prescription profiles and the personal characteristics of the doctor or characteristics of the health unit. The prescribing patterns of the 95 doctors from 12 health units showed considerable variability (for alprazolam DUD: mean=19.7, standard deviation $(S D)=9.3$, minimum $=5.1$, maximum=60.8; for fluoxetine DUD: mean=9.2, $\mathrm{SD}=4.4$, minimum $=0.7$, maximum $=28.4$ ). High prescribers of alprazolam (above the 95 th percentile), prescribed around 5 times more than lower prescribers (below the 5 th percentile). The same was found regarding fluoxetine prescribing. Most doctors prescribed 60 tablets of alprazolam at a time.

Conclusion: Results are comparable to other studies, reinforcing the potential of using the available SIARS $\odot$ tool to study prescriptions. The observed variability highlights the necessity to adapt SIARS $\odot$ to the internationally recognized unit, number of DUD, that will allow meaningful comparisons and quality development measures.

Keywords: Family Physicians; Drug Prescriptions; Psychotropic Drugs. 